\title{
Numerical Simulation of a Cod End Net Part 1: Equilibrium in a Uniform Flow
}

\author{
H. Le Dret ${ }^{1}$, R. Lewandowski ${ }^{2}$, D. Priour ${ }^{3 *}$ and F. Chagneau ${ }^{4}$ \\ ${ }^{1}$ Laboratoire Jacques-Louis Lions, Université Pierre et Marie Curie, boîte courrier 187, 75252 Paris Cedex 05, \\ France \\ ${ }^{2}$ IRMAR, Université de Rennes I, Campus Beaulieu, 35042 Rennes Cedex, France \\ ${ }^{3}$ IFREMER, B.P. 70, Pointe du Diable, 29280 Plouzané Cedex, France \\ ${ }^{4}$ IUT de Rennes, rue du Clos Courtel, B.P. 90422, 35704 Rennes Cedex 7, France \\ *: Corresponding author : daniel.priour@ifremer.fr
}

\begin{abstract}
In this paper, a cod-end fish net is modelled by a discrete finite network. The nonlinear elastic energy for such a structure is defined and studied. Numerical simulations of the equilibrium state are performed by minimizing the total energy. The minimization algorithm uses a general nonlinear conjugate gradient procedure.
\end{abstract}

Keywords: elastic networks - nonlinear conjugate gradient - numerical simulation 


\title{
Numerical Modeling and Simulation of a Cod End Net Using a Discrete Nodal Model. Part 1: Equilibrium in a Uniform Flow
}

\author{
H. Le Dret* R. Lewandowski ${ }^{\dagger}$ D. Priour ${ }^{\ddagger}$ and F. Chagneau ${ }^{\S}$
}

\begin{abstract}
In this paper, a cod-end fish net is modelled by a discrete finite network. The nonlinear elastic energy for such a structure is defined and studied. Numerical simulations of the equilibrium state are performed by minimizing the total energy. The minimization algorithm uses a general nonlinear conjugate gradient procedure.
\end{abstract}

\section{Introduction}

\subsection{General background}

The efforts expended by the fishing industry to achieve a sustainable activity require an improvement of the knowledge of fishing gear behavior at sea, especially that of the netting.

Figure 1.1: Underwater view of cod-end netting; empty cod-end

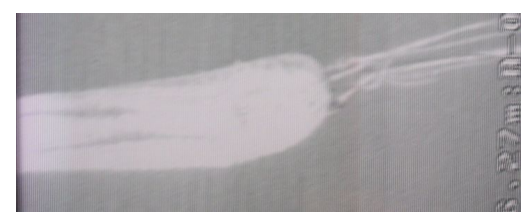

In fact, netting performance in catching fish is directly linked to the shape of the netting under water. The only ways to observe this shape are

i) observations at sea,

ii) tests in a flume tank on a mock-up and

${ }^{*}$ Laboratoire Jaques-Louis Lions, Université Pierre et Marie Curie, boîte courrier 187, 75252 Paris Cedex 05, France, ledret@ccr.jussieu.fr

$\dagger$ IRMAR, Campus Beaulieu, Université de Rennes I, 35042 Rennes Cedex, France, Roger.Lewandowski@univ-rennes1.fr, http://name???.math.univ-rennes1.fr/

ॠIFREMER, BP 70, Pointe du Diable 29280 Plouzané Cedex, France, daniel.priour@ifremer.fr

$\S$ IUT de Rennes, rue du Clos Courtel, BP 90422, 35704 Rennes Cedex 7, France 
iii) numerical simulations.

i) Observations at sea are limited because of the visibility, which only reaches a few meters when the netting is dragged at depths of a few hundred meters. The photographs above and below (see figure 1.1 and figure 1.2) show first an empty cod-end net, then a cod-end net with trapped fish.

Figure 1.2: Underwater view of cod-end netting with trapped fish

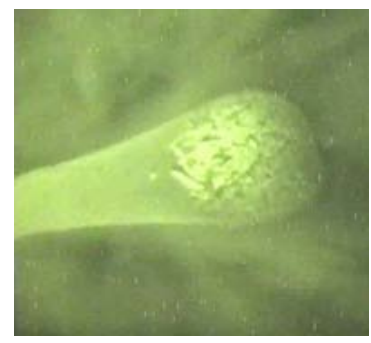

These photographs were taken by the IFREMER EROC remote controlled submarine, and are presented here courtesy of IFREMER.

ii) Flume tank tests are based on scaled models, which do not exactly reflect the netting properties, see [10].

Figure 1.3: View of a cod-end in a flume tank; the fish is replaced by plastic bags filled with water

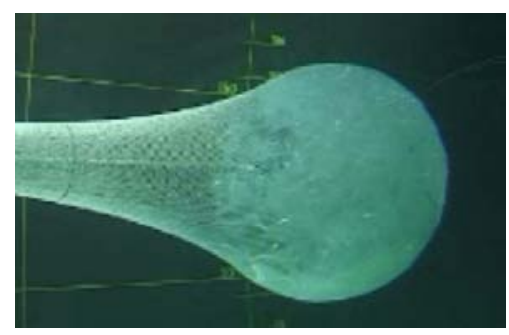

Notice that in each case, pictures 1.2 and 1.3 show that the net end takes on an ellipsoidal aspect when full.

iii) Numerical simulations do not present the above mentioned drawbacks and show a lot of promise due to ever increasing available computing power. Such numerical simulations are the subject of this paper.

Two main types of netting meshes are used in the fishing industry-diamond or square meshes and hexagonal meshes. Diamond or square meshes are a lot more commonly used than hexagonal meshes. They are made of two directions of twines. Generally speaking the twines are of the same length in the unstretched state.

Hexagonal meshes are made of three directions of twines. Due to manufacturing considerations, the three twines are not always of the same length in the unstretched state. Some diamond meshes with very long twine seams could be considered as hexagonal meshes. 
Figure 1.4: Piece of diamond mesh net at rest

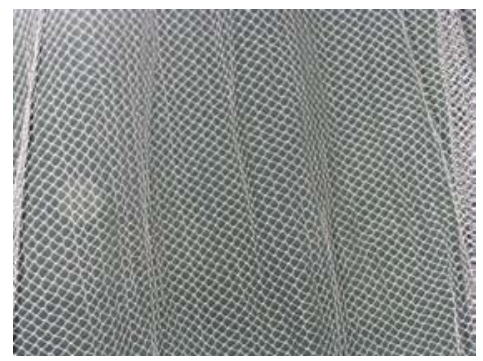

In the numerical part of what follows, we will only study nettings made of diamond meshes. Hexagonal meshes are studied in [11] and [12].

\subsection{Motivations}

The long term goal of this study is to provide a numerical code capable of simulating the coupling between the net, the flow and the fish. Naturally, several tools already exist. There are codes that compute the deformations of nets under given external forces. There also are simulations using FLUENT code that compute the flow in axisymmetric rigid cod-ends, without fish nor net deformation and fluid/structure interaction. The reader will get a precise idea of the sate of the art at PREMECS project website

\section{http://www.ifremer.fr/premecs/}

After looking at the bibliography we note that

- The only rigorous mathematical concept for discrete networks is to be found in the work of Rivlin [16] in the case of inextensible twines. This study was extended to extensible cords by Green and Shi [6] and [7]. The work of the latter author was motivated by issues pertaining to the textile industry. It is more concerned with continuous, membranes models and cannot be used directly in our problem.

- The mathematical problem of the interaction of the net with the flow does not seem to have ever been properly posed. A very good physical analysis can be found in the Ph.D. thesis by Theret [18]. This author clearly describes the interaction between a constant flow with a cable in the inextensible case. There are many experimental results that we will use in the following.

- We are not aware of any numerical simulations using finite elements of a net immersed in a flow and deforming under flow interaction. It is know that finite elements is the best numerical method for fluids (see [13]), in particular in the case on complex geometries and also in the case of coupling fluids and structures (see also [8]).

- Again, we are not aware of any numerical simulations including bodies representing the fish getting trapped in the net by the flow, and interacting with the net. 
Our main motivation is to try and fill the above mentionned gap, by formulating a rigorous mathematical problem and creating a complete code in $\mathrm{C}++$ to simulate the behavior of the full net/flow/fish system.

Naturally, we will proceed in progressive stages. The main goal of the present part one is:

- to give a rigorous mathematical concept of a discrete network by a nodal definition,

- to define the equilibrium state of the net by minimizing the total energy, which is comprised of the elastic energy and external potentials acting on the net.

- to obtain an equilibium state which fits with observations of net in flows (see figure 1.1 and 1.2). Numerical minimizers will be obtained using a nonlinear conjugate gradient algorithm.

- the fluid/fish/net interaction problem will be the subject of forthcoming work. Let us just note that the equilibrium net configurations obtained here will be used in this forthcoming work as initial data for the evolution problem of an immersed net gradually filling with balls that represent the fish.

\subsection{Outline of the paper}

In view of figure 1.4, due to the large number of twines combined with a small period, it could be tempting to model a net using a homogenized, 2D continuum mechanics model. We do not choose this route, however, because direct modeling and simulation in terms the individual twines is perfectly amenable to standard computers. There is thus no need to add an extra mathematical modeling step before going to numerical experiments. Note that there are mathematical studies for very similar situations in the context of atomic to continuum model transition, see [3].

We thus first give a rigorous mathematical definition of our net model and how it deforms under the action of applied forces. The net is geometrically modelled by the position of its knots in three-dimensional space, which we call the nodes. An abstract connection matrix is defined that represents the twines of the net. In this sense, the net is identified with the position of its node and the connection graph that is naturally associated with the twines. Deformations of the net are described by the Lagragian deplacement of the nodes. In section 2 below, we give a precise definition of the mathematical object that we use in the numerical code. This definition assume that:

- Each twine in extension is assumed to remain straight and uniformly stretched throughout the deformation. No assumption is made concerning unstretched twines.

- During deformation, twines only interact through their common nodes.

These hypotheses seem to fit well with the observations (see figure 1.2 and 1.3). The second hypothesis means that we preclude situations in which the net could become entangled. Note that the Lagrangian deformation/net topology description we adopt here is consistent with standard mesh formats (in the sense of scientific computing), which 
makes it possible to export computation results to such mesh visualization tools as medit (http://www-rocq1.inria.fr/gamma/medit/medit.html).

Next, we define a general nonlinear elastic energy for the net. This energy is derived from the elastic energy for each twine. It is by construction frame-indifferent, which is good from the mechanical point of view, and therefore highly nonlinear, hence its numerical treatment will be computationally intensive. In the simulations, we will use a cubic nonlinear constitutive law for each twine, corresponding to a quartic twine energy. This nonlinear law was obtained using the results of traction experiments carried out on a twine sample provided by IFREMER (see section 4). Note that retaining a linear constitutive law for each twine would only marginally affect the nonlinearity of the global energy. We will come back to that later.

The drag and lift forces exerted on the net by the flow are not conservative forces. We model them here by dead loadings that fit the expected values of these forces at equilibrium both in terms of magnitude and direction in three-dimensional space. The forthcoming evolution simulations will use more realistic dissipative forces. We adopt the simplified hypothesis of a uniform flow inside and around the net, as is done for instance in [18], where the hypothesis of Landweber is used. We also use Landweber type hypotheses to model lift and drag forces in our code. The resulting equilibrium shapes are perfectly acceptable.

This hypothesis of uniform flow inside the net is questionable in terms of fitting with reality. Unfortunately, as emphasized in [18], several different experiments lead to contradictory results concerning the nature of the flow inside and near the net. It certainly depends on the nature of the net, the size of the meshes, the diameter of the twine and many other parameters. We plan to address this question via other numerical experiments.

The equilibrium state of the net is achieved by minimizing the total energy, which is nonlinear. To do that, we use the classical conjugate gradient method (see [4] for the quadratic case) adapted to the nonlinear case. The algorithm we chose is due to PolakRibière (see subsection 3.3 in the remainder of the article).

Acknowledgements This work has been supported by IFREMER, under contract 200322030299.

\section{The general mathematical elastic net model}

\subsection{Orientation}

In this section, a rigorous, formal mathematical definition of a cod-end net and the associated elastic energy under deformation is given. In spite of the fact that the present article is not intended as a purely mathematical article but as a numerical article, these definitions form the basis of the code we developed and therefore are useful in understanding our numerical approach. These definitions entail many mathematical questions, which are currently under consideration and will be the subject of another article.

\subsection{Geometrical and topological definition of a net with basis $\Gamma$}

Our global strategy to model nets hinges on the following definition. 
Definition 2.1 Let $\Gamma$ be a closed Jordan curve in $\mathbb{R}^{3}, \mathcal{N}=\left\{P_{1}, \ldots, P_{N}\right\} \subset\left(\mathbb{R}^{3}\right)^{N}$ and $A \in \mathbb{M}_{N}(\mathbb{R})$ a symmetric $N \times N$ matrix. We say that the couple $(\mathcal{N}, A)$ is a N-net with basis $\Gamma$ of order $q_{0}\left(q_{0}<N\right)$ if and only if:

1) For all indices $1 \leq i \leq q_{0}$, the point $P_{i}$ belongs to $\Gamma$.

2) The matrix $A=\left(a_{i j}\right) \in \mathbb{M}_{N}(\mathbb{R})$ is a graph connection matrix, i.e., $a_{i j}=0$ or $a_{i j}=1$ for every $1 \leq i, j \leq N$.

We say that it is a diamond-shaped net if, in addition,

3) (2 connections for boundary nodes) For every $1 \leq i, j \leq q_{0}, a_{i j}=0$ and for all $1 \leq i \leq q_{0}$, there exist exactly two indices $k_{i} \neq q_{i}$ such that $a_{i k_{i}}=a_{i q_{i}}=1$.

4) (4 connections for interior nodes) For all $q_{0}<i \leq N$, there exist exactly four indices $k_{i}, q_{i}, r_{i}$ and $s_{i}$ such that $a_{i k_{i}}=a_{i q_{i}}=a_{i r_{i}}=a_{i s_{i}}=1$, except possibly for one index.

5) (diamond-shaped condition) Let $i, j$ be such that $a_{i j}=1$ and not the exceptional index of point 4 . If $i, j>q_{0}$, there exist exactly two couples of indices $\left(k_{i}^{+}, k_{j}^{+}\right)$and $\left(k_{i}^{-}, k_{j}^{-}\right)$ such that

$$
a_{i k_{i}^{ \pm}}=a_{j k_{j}^{ \pm}}=a_{k_{i}^{ \pm} k_{j}^{ \pm}}=1 \quad \text { and } \quad a_{j k_{i}^{ \pm}}=a_{i k_{j}^{ \pm}}=0 .
$$

If $i \leq q_{0}$ or $j \leq q_{0}$, the same holds with exactly one couple of indices.

Comments 2.1 The intended meaning of point 2 in the above definition is that when $a_{i j}=1$, the nodes $P_{i}$ and $P_{j}$ are connected by a twine and when $a_{i j}=0$ they are not connected by a twine. We use the convention that $a_{i i}=0$.

The introduction of the connexion matrix $A$ makes for a convenient description of the topology of the net. In particular, it is possible to describe in this way a net that is physically composed of several parts, some diamond-shaped and others hexagonal, or even more general topologies. All this information is encoded in the symmetric matrix $A$.

The intended meaning of point 3 is that boundary nodes are only attached to two distinct interior nodes and the intended meaning of point 4 is that interior nodes are attached to four distinct interior or boundary nodes. The exceptional node in point 4 is there to account for the presence of the cod-end node were many twines can come together.

Points 4 and 5 together embody the fact that the nodes/twines in a diamond-shaped net are topologically arranged in lozenges.

The mathematical description of the physical twines is now a simple byproduct of the definition of the net.

Definition 2.2 Let $\mathbf{u}_{i j}$ be the vector in $\mathbb{R}^{3}$ originating at $P_{i}$ and ending at $P_{j}$. We say that $\mathbf{u}_{i j}$ is a twine of the net $(\mathcal{N}, A)$ if and only if $a_{i j}=1$. For such indices, we let

$$
l_{i j}^{0}=\left\|\mathbf{u}_{i j}\right\|,
$$

to be the length of the corresponding twine.

Comments 2.2 Note that each physical twine is described by two vectors $\mathbf{u}_{i j}$ and $\mathbf{u}_{j i}$. The (incomplete) matrix $\left(l_{i j}^{0}\right)$ may be used to encode information pertaining to the natural, unstretched length of the twines. In a similar way as for the topology of the net, we can thus conveniently describe nets with varying twine length not only within a single mesh, but globally with some parts of the net, i.e., some index couples, having much larger twine lengths than other parts of the net. 


\subsection{Net deformations}

In what follows, we consider a given net $(\mathcal{N}, A)$ with basis $\Gamma$ that we fix until the end of the section. In Lagrangian parlance, we take this net as reference configuration from which deformations are measured. Note that this is an arbitrary choice, but that some nets may have particular reference configurations of interest, depending on their global topology. For example, a rectangular pane of diamond-shaped netting can be arranged in a planar configuration where all twines are at their natural length and all lozenges are translates of each other, as in figure 1.4.

Let

$$
\mathbf{H}(\mathcal{N})=\left\{\beta: \mathcal{N} \rightarrow \mathbb{R}^{3}\right\}
$$

Since the net is a finite set, $\mathbf{H}(\mathcal{N})$ can be identified with $\left(\mathbb{R}^{3}\right)^{N}$, hence it is finite dimensional and therefore locally compact.

We want to model the fact that the net is rigidly attached to some support on the nodes that belong to the basis. In fact, this support usually is another piece of netting, but we ignore this for the time being.

Definition 2.3 Let $\beta \in \mathbf{H}(\mathcal{N})$. We say that $\beta$ is an admissible deformation for the net $(\mathcal{N}, A)$ if and only if for all $1 \leq i \leq q_{0}, \beta\left(P_{i}\right)=P_{i}$. We denote by $\mathbf{V}(\mathcal{N})$ the set of all admissible deformations.

We note that the set $\mathbf{V}(\mathcal{N})$ is a closed subset of $\mathbf{H}(\mathcal{N})$ hence also locally compact. The following proposition is quite obvious.

Proposition 2.1 For all admissible deformations $\beta \in \mathbf{V}(\mathcal{N})$, the couple $(\beta(\mathcal{N}), A)$ is $N$ net with basis $\Gamma$ of order $q_{0}$. In addition, if $(\mathcal{N}, A)$ is diamond-shaped, so is $(\beta(\mathcal{N}), A)$.

We now introduce a convenient, albeit slightly incorrect notation.

Definition 2.4 For $\beta \in \mathbf{H}(\mathcal{N})$ we note $\beta\left(\mathbf{u}_{i j}\right)$ the vector in $\mathbb{R}^{3}$ originating at $\beta\left(P_{i}\right)$ and ending at $\beta\left(P_{j}\right)$.

If $a_{i j}=1,\left\|\beta\left(\mathbf{u}_{i j}\right)\right\|$ is the length of the corresponding deformed twine.

\subsection{Total net energy and minimization problem}

Let us now switch to the mechanical part of the modeling process. Let us first recall our two basic hypotheses.

1. Each twine in extension is assumed to remain straight and uniformly stretched throughout the deformation. No assumption is made concerning unstretched twines.

2. During deformation, twines only interact through their common nodes. 
Twines are made of synthetic fibers. Such synthetic materials are adequately described by hyperelasticity, that is to say nonlinear three-dimensional elasticity with an elastic energy, in their elastic regime, see [5]. Moreover, it is possible to give a rigorous mathematical derivation of nonlinearly elastic one-dimensional string and wire models from three-dimensional hyperelasticity, see [1]. It is therefore reasonable to ascribe to each deformed twine an elastic energy of a form that is compatible with that obtained from such derivations, namely

$$
e_{i j}=W_{i j}\left(\left\|\beta\left(\mathbf{u}_{i j}\right)\right\| / l_{i j}^{0}\right) l_{i j}^{0},
$$

where $W_{i j}$ is a $C^{1}$-function from $\mathbb{R}^{+}$into $\mathbb{R}$ that can be computed from the three-dimensional stored energy function. Note that the twine energy depends only on the twine deformation gradient $\beta\left(\mathbf{u}_{i j}\right) / l_{i j}^{0}$ (the straightness and uniform stretching hypothesis is used here) via its norm, due to material frame indifference.

It can be shown that if $l_{i j}^{0}$ is the natural length of the twine, which we will assume from now on, then $W_{i j}(t)=0$ for $0 \leq t \leq 1$, see [1]. Mathematically speaking, this is a relaxation effect related to the convexification of the energy. From the mechanical point of view, this property means that twines in compression are lax and thus contribute no energy nor tension. This is only approximately true in our case since there are flexural and torsional effects in compression, but these are negligible with respect to tension effects in a first analysis.

Let now $\beta \in \mathbf{V}(\mathcal{N})$ be an admissible deformation. This deformation will naturally have a total elastic energy

$$
E(\beta)=\sum_{(i, j) \in \mathcal{J}} W_{i j}\left(\left\|\beta\left(\mathbf{u}_{i j}\right)\right\| / l_{i j}^{0}\right) l_{i j}^{0},
$$

where $\mathcal{I}$ is the set of indices $i<j$ such that $a_{i j}=1$. Note that this energy can also be written as

$$
E(\beta)=\frac{1}{2} \sum_{i, j=1}^{N} a_{i j} W_{i j}\left(\left\|\beta\left(\mathbf{u}_{i j}\right)\right\| / l_{i j}^{0}\right) l_{i j}^{0},
$$

which is mathematically convenient, but not computationally efficient. The energy thus depends on the net topology via the graph connection matrix $A$.

We next assume that the net nodes are submitted to conservative forces, which are described by $C^{1}$ potentials $\Phi_{i}: \mathbb{R}^{3} \rightarrow \mathbb{R}$. The corresponding potential energy of the deformed net is thus

$$
F(\beta)=\sum_{i=1}^{N} \Phi_{i}\left(\beta\left(P_{i}\right)\right) .
$$

The simplest possible potential corresponds to the case of dead loadings

$$
\Phi_{i}(P)=f_{i} \cdot O P
$$

where $f_{i} \in \mathbb{R}^{3}$ represents the dead force applied at point $P_{i}$.

The total net energy corresponding to an admissible deformation $\beta$ is then given by

$$
\begin{aligned}
I(\beta) & =E(\beta)-F(\beta) \\
& =\frac{1}{2} \sum_{i, j=1}^{N} a_{i j} W_{i j}\left(\left\|\beta\left(\mathbf{u}_{i j}\right)\right\| / l_{i j}^{0}\right) l_{i j}^{0}-\sum_{i=1}^{N} \Phi_{i}\left(\beta\left(P_{i}\right)\right),
\end{aligned}
$$


and stable equilibrium configurations are obtained by minimizing the total energy over the set of admissible configurations, that is to say solving the variational problem: find $\beta_{*} \in \mathbf{V}(\mathcal{N})$ such that

$$
I\left(\beta_{*}\right)=\inf _{\beta \in \mathbf{V}(\mathcal{N})} I(\beta) .
$$

Since this is a finite dimensional problem and the energy is continuous, this problem will have at least one solution as soon as $I(\beta) \rightarrow+\infty$ when $\|\beta\| \rightarrow+\infty$, which is ensured by assuming enough coercivity for the elastic energy with respect to the force potentials, and by the boundary condition of place on $\Gamma$. This will be the case in the exemples considered below.

If $\beta_{*}$ is an energy minimizer, then it is a solution of the associated Euler-Lagrange equations

$$
\nabla_{P} E\left(\beta_{*}\right)=\nabla_{P} F\left(\beta_{*}\right) .
$$

In mechanical terms, this is nothing but the equality of resultant elastic forces on the lefthand side with applied forces on the right-hand side. These equations are thus ipso facto solved by the minimization problem.

Let us now focus on various possible choices for the elastic energy.

\subsection{Linearly elastic energy}

In what follows, we will assume for simplicity that all twines are made of the same material and have the same mechanical characteristics. Hence there is only one elastic energy density $W: \mathbb{R}_{+} \rightarrow R$ such that $W_{i j}=W$ for all $i$ and $j$ describing all twines.

The simplest twine elastic energy density corresponds to linear elasticity. It is thus essentially quadratic, but needs to be truncated in compression as explained above. Such truncation is also a classical hypothesis in the present context (see for instance in [7], [6] and [17]). Denoting by $u_{+}=\max (u, 0)$ the positive part of $u$, we have in this case

$$
W(t)=\frac{k}{2}\left((t-1)_{+}\right)^{2},
$$

where the stiffness coefficient $k$ depends on the material. In the case of an isotropic, homogeneous material, we have $k=E A$ where $E$ is the Young modulus of the bulk material and $A$ the twine cross-section area.

The linearly elastic choice thus leads to a total elastic energy of the form

$$
E(\beta)=\sum_{(i, j) \in \mathcal{g}} \frac{k}{2 l_{i j}^{0}}\left(\left(\left\|\beta\left(\mathbf{u}_{i j}\right)\right\|-l_{i j}^{0}\right)_{+}\right)^{2} .
$$

Note that this function is $C^{1}$ and coercive over $\mathbf{V}(\mathcal{N})$. Moreover, even if the context is that of linear elasticity, the net energy is nonetheless highly nonlinear, due to its dependence on the norm of $\beta\left(\mathbf{u}_{i j}\right)$, which is required by material frame indifference, and the positive part. There is thus no escaping nonlinearity. 


\subsection{Nonlinear corrections}

Further numerical experiments concerning the interaction of floating rigid bodies with the net, not presented in this paper, indicate that the linear constitutive law above is not sufficient to prevent such a body from easily stretching a single mesh and passing through the net. This undesirable behavior can be avoided by adding nonlinear correction terms. It should be noted that these nonlinear corrections do not significantly add to the theoretical and computational complexity of the problem, since it is already nonlinear in the simplest possible setting.

Moreover, as we will see further on (see section 4), experimental data clearly indicate that the linearly elastic range of twines is not that large and that nonlinear effects occur rather rapidly. Therefore, we are led to use a nonlinear elastic density of the form

$$
W(t)=\sum_{p=1}^{n} \frac{k_{p}}{p+1}\left((t-1)_{+}\right)^{p+1},
$$

that correspond to a total elastic net energy of the form

$$
E(\beta)=\sum_{(i, j) \in \mathcal{J}} \sum_{p=1}^{n} \frac{k_{p}}{(p+1)\left(l_{i j}^{0}\right)^{p}}\left(\left(\left\|\beta\left(\mathbf{u}_{i j}\right)\right\|-l_{i j}^{0}\right)_{+}\right)^{p+1} .
$$

Subsection 4.2 shows that a very good approximation of experimental data is achieved for $n=3$. The stiffness coefficients $k_{p}$ are computed in this subsection.

\section{Equilibrium in the specific case of the empty cod-end}

\subsection{Cod-end topology}

It is more easily explained with pictures than with its connection matrix. We start from a square pane of diamond-shaped netting as follows

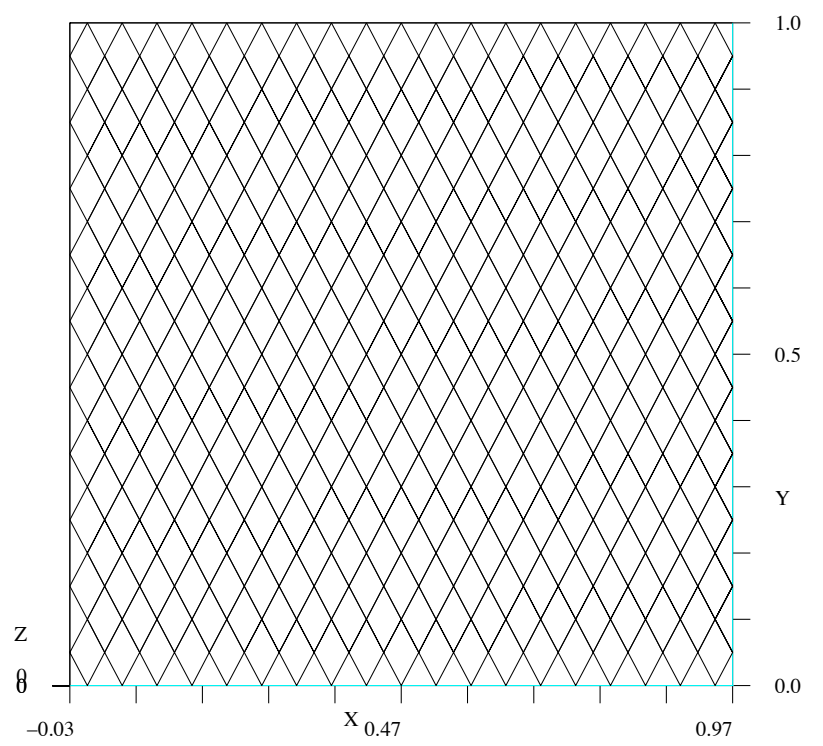


In this example, there are 19 meshes horizontally and 10 meshes vertically, which correspond to horizontal rows of 20 or 19 nodes depending on parity and vertical (staggered) rows of 21 nodes. We then map this square into a circle via a Cartesian to polar coordinates transform: the $x$ coordinate becomes the radial coordinate and the $y$ coordinate becomes the angular coordinate.

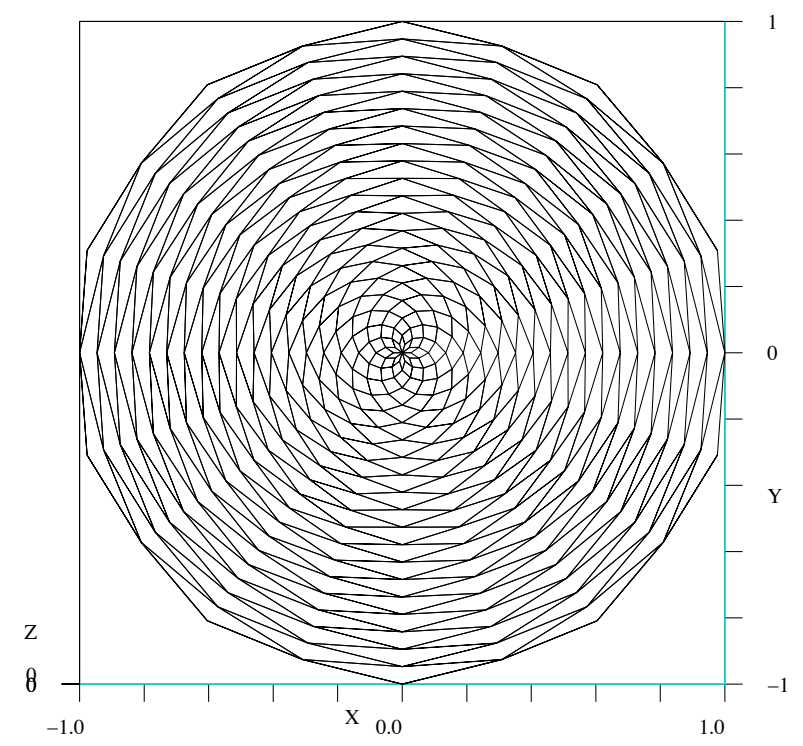

The resulting circular net thus has 10 meshes around and 19 meshes from the center to the external edge. Note that the central node has more than 4 connections. It corresponds to the cod-end. This flat circular configuration can be used as a reference configuration for energy minimization, but we will see that 3D configurations will be more appropriate for realistic computations. There is no theoretical difference between the two, however, in the Lagrangian formalism.

The global effect is the same as taking two or more rectangular panes, attaching them together by their sides along boltropes and then tying the bottom nodes together to form the cod-end.

\subsection{Modeling drag and lift}

The net is placed in a uniform current. We assume that buoyancy is compensated by gravity. Thus, when the net contains no catch, it is only submitted to the forces due to the flow, namely drag and lift. The Landweber and Plotter hypotheses to compute the drag force due to the flow asserts that the drag does not depend on the angle made by two twines issuing from the same node[9]. Under these hypotheses, the drag force acting on a twine $\mathbf{u}_{i j}$ of current length $l_{i j}=\left\|\beta\left(\mathbf{u}_{i j}\right)\right\|$ (see definitions 2.1, 2.2 and 2.4 above) is constant and given by

$$
\mathbf{F}_{i j}=\frac{1}{2} f \rho C_{d} d l_{i j} \mathbf{U}\|\mathbf{U}\|
$$


where $\mathbf{U}$ is the water speed (constant and equal to $1 \mathrm{~m} / \mathrm{s}$ horizontally in the simulation below), $f$ is the friction coefficient of the twine-according to [18] $f=0.08, \rho$ is the water density $\left(10^{3} \mathrm{~kg} / \mathrm{m}^{3}\right), d$ is the diameter of a twine, typically $3 \mathrm{~mm}$ or $4 \mathrm{~mm}$, and $C_{d}$ is the drag coefficient of the twine, 1.2 or 1.8 according to [2].

Due to the fact that the lengthening of each twine is small, we will make the following the reasonable simplified approximation:

$$
\mathbf{F}_{i j}=\frac{1}{2} f \rho C_{d} d \ell_{i j}^{0} \mathbf{U}|\mathbf{U}|
$$

When distributed over the nodes, this force is a dead load.

There are similar hypotheses for the lift, but the latter should depend on the angle between the twine and the flow, and is therefore not a dead load. To simplify computations here, we chose to model the lift force using a dead load that acts radially outwards on the disk and of constant norm computed from similar formulas as (3.2).

\subsection{The nonlinear conjugate gradient method: the Polak-Ribière al- gorithm}

To compute the minimizer of the total energy $I$ over the set of admissible deformations, we use the conjugate gradient algorithm for a non quadratic functional. There are several versions of this algorithm, we use the Polak-Ribière version which is usually the preferred version. A simple presentation can be found at http://csep1.phy.ornl.gov/mo/node20.html. The fundamental mathematical principles for the conjugate gradient procedure can be found in [4] in the case of a quadratic functional. Let us recall that the algorithm takes the following form. We start with an initial deformation $\beta_{0}=I d$ (not necessarily the reference configuration) and compute a first descent direction $d_{0}=-\nabla I\left(\beta_{0}\right)$. Then, the iterative procedure is given by

$$
\begin{aligned}
& g_{k}=\nabla I\left(\beta_{k}\right) \\
& d_{k}=-g_{k}+B_{k} \beta_{k-1} \\
& \beta_{k+1}=\beta_{k}+\lambda_{k} d_{k}
\end{aligned}
$$

where the coefficients $\lambda_{k}$ and $B_{k}$ are computed by the following formulae:

$$
\begin{aligned}
& I\left(\beta_{k}+\lambda_{k} d_{k}\right)=\min _{\lambda \in \mathbb{R}} I\left(\beta_{k}+\lambda d_{k}\right) \\
& B_{k}^{P R}=\frac{g_{k}^{T}\left(g_{k}-g_{k-1}\right)}{g_{k_{1}}^{T} g_{k-1}} \\
& B_{k}=\max \left(B_{k}^{P R}, 0\right) .
\end{aligned}
$$

As opposed to the case of a quadratic functional, for which the iteration is entirely explicit and is theoretically guaranteed to exactly yield the minimizer in a number of steps that is at most the dimension of the space, round-off errors and ill-conditioning problems notwithstanding, the non quadratic algorithm spends most of its time computing the scalar $\lambda_{k}$. This computation requires the minimization of a function in one variable, 
Figure 4.1: Lloyd EZ 20 and sample
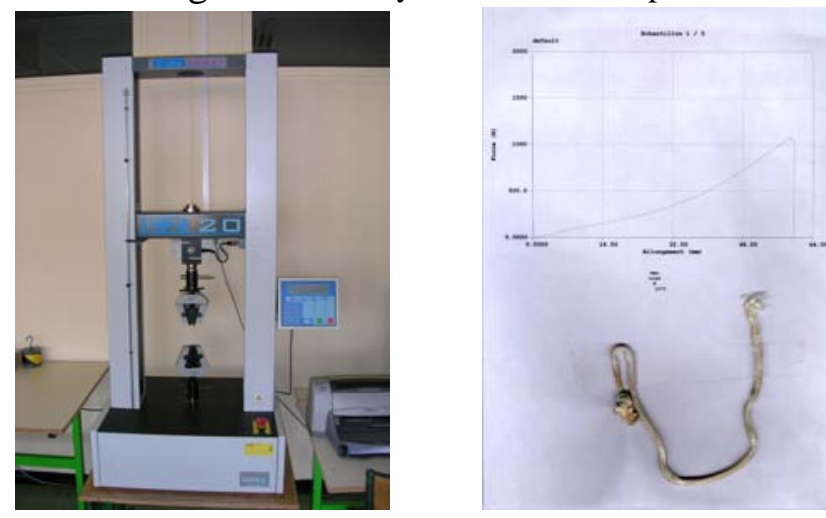

which is classically achieved by using a combination of golden rule trisection and inverse parabolic interpolation. This step in the algorithm a priori requires many evaluations of the function $I$ and is the most costly of all.

The algorithm is robust but may entail a large number of iterations. It is thus a good idea to use as starting point $\beta_{0}$ a configuration that is likely to be close to the minimizer. This is not the case of the flat circular configuration described above.

\section{Experimental results for the stiffness of a twine}

\subsection{Experimental data}

The elastic energy of a twine is not directly accessible, but the traction force can be measured. In other words, it is possible to experimentally determine the constitutive law of the twine, i.e., the strain-stress law. Since this is a one-dimensional situation, the energy density follows by integration of the strain-stress law.

Traction experiments were performed using the EZ 20 Lloyd machine (see figure 4.1). The twine sample used was from a net provided courtesy of Ifremer. The twine under consideration was made of a braided polyamide of diameter ... $\mathrm{mm}$.

The EZ 20 Lloyd machine graphs the twine stretch as a function of the applied force. The applied force is longitudinal.

The graphed variables are

$$
x=\frac{\Delta \ell}{\ell_{0}}, \quad \ell_{0}=0.27 \mathrm{~m}, \quad y=T,
$$

where $\ell_{0}$ is the length of the sample at rest, $\Delta \ell$ the stretching, $T$ the applied tension measured in Newtons (Tab.1, Fig. 3)). 
Figure 4.2: Experimental twine stiffness curve

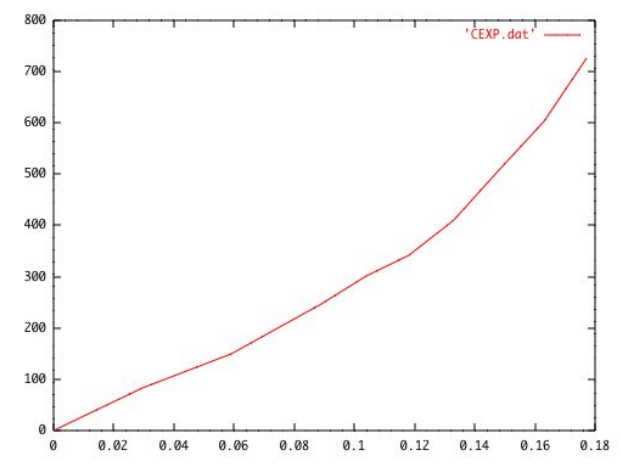

Table 1: Twine stiffness, $\mathrm{x}$ strain, $\mathrm{y}$ tension $(\mathrm{N})$

$$
\left[\begin{array}{ll}
x & y \\
x_{0}=0 & y_{0}=0 \\
x_{1}=0.0296 & y_{1}=82.2 \\
x_{2}=0.0592 & y_{2}=150.7 \\
x_{3}=0.0888 & y_{3}=246.6 \\
x_{4}=0.104 & y_{4}=301.4 \\
x_{5}=0.118 & y_{5}=342.5 \\
x_{6}=0.133 & y_{6}=411.0 \\
x_{7}=0.148 & y_{7}=506.9 \\
x_{8}=0.163 & y_{8}=602.8 \\
x_{9}=0.177 & y_{9}=726.1
\end{array}\right]
$$

This curve is obviously nonlinear. Nevertheless, it is quite commonly accepted that the tension applied on each twine is small enough to remain in the linear elasticity range (see [15], [14]). This is true when only drag and lift corresponding to speeds of the order of $1 \mathrm{~m} / \mathrm{s}$, which are rather small forces, are present, but we have already seen that problems may arise when the interaction of the net with rigid bodies is considered. Moreover, adding nonlinear terms does not essentially complicate the model, nor does it significantly raise computational costs. Let us also mention that net models with inextensible twines can also be considered (see [16], [11] and [12]).

The above experimental data in the case of the considered sample suggest that the linear part of the constitutive law is (Fig. 4)

$$
T=2770 \frac{\Delta \ell}{\ell_{0}} .
$$

This constitutive law corresponds to the energy density

$$
W(t)=\frac{2770}{2}\left((t-1)_{+}\right)^{2} .
$$


Figure 4.3: Experimental curve and linear approximation

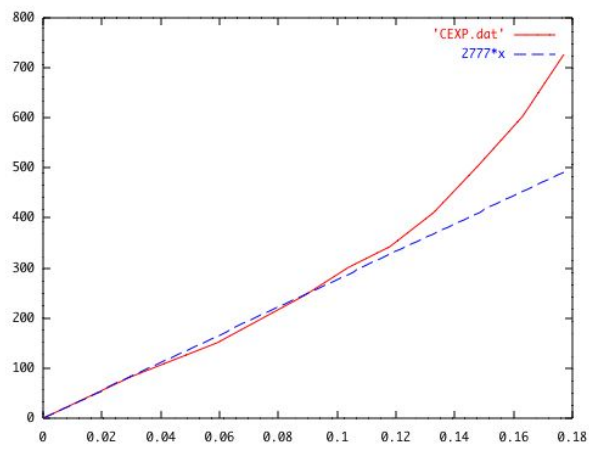

Figure 4.4: Experimental curve and polynomial law of order 3

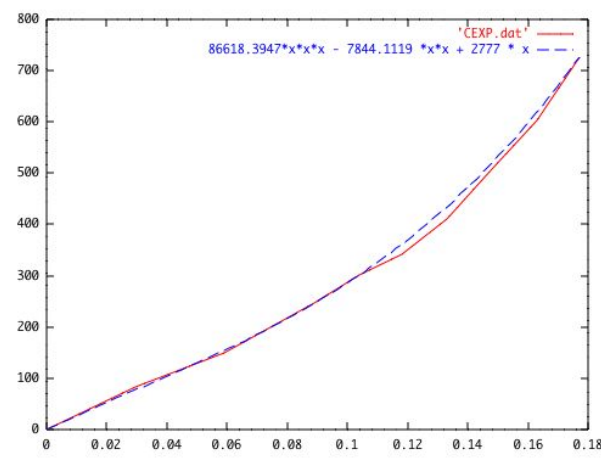

\subsection{Nonlinear correction of order 3}

We are looking for a more refined law of the form

$$
T=k_{1} \frac{\Delta \ell}{\ell_{0}}+k_{2}\left(\frac{\Delta \ell}{\ell_{0}}\right)^{2}+k_{3}\left(\frac{\Delta \ell}{\ell_{0}}\right)^{3}+\cdots
$$

A second order correction obtained either by the least square method or by interpolation does not give satisfactory results, thus we go directly to third order correction.

The linear law obtained above suggests that we should take $k_{1}=2777$. Coefficients $k_{2}$ and $k_{3}$ are then conveniently determined by simple interpolation on two points of the experimental graph. We find the following law

$$
T=2780 \frac{\Delta \ell}{\ell_{0}}-7840\left(\frac{\Delta \ell}{\ell_{0}}\right)^{2}+86600\left(\frac{\Delta \ell}{\ell_{0}}\right)^{3},
$$

a law which fits very well with the experimental curve, as can be seen on Figure 4.4.

This constitutive law corresponds to the energy density

$$
W(t)=\frac{2770}{2}\left((t-1)_{+}\right)^{2}-\frac{7840}{3}\left((t-1)_{+}\right)^{3}+\frac{86600}{4}\left((t-1)_{+}\right)^{4} .
$$


Figure 5.1: Conical configuration

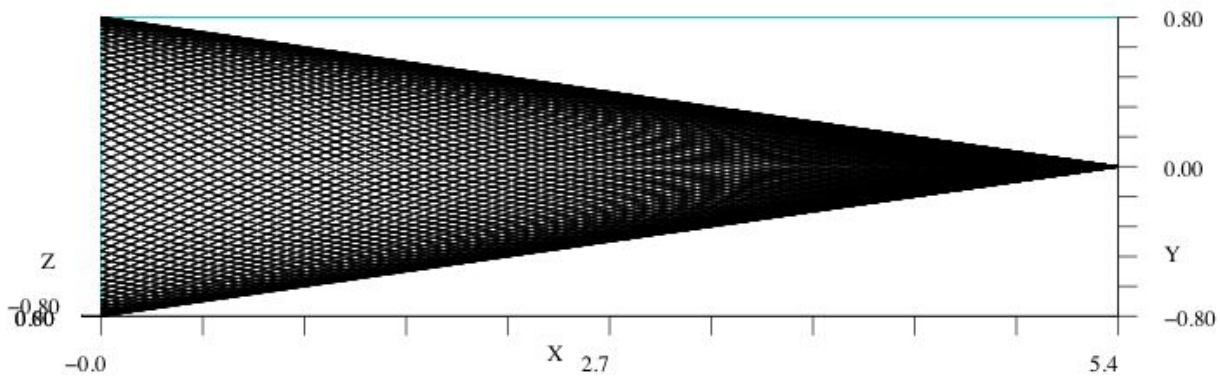

Note that the third order law (4.5) is really a perturbation of the linear law (4.3). Its coefficients are large, which indicate that the twine is quite stiff. We thus do not expect much stretching to occur under the sole action of the drag and lift forces.

\section{Numerical results}

The code was developed using Scilab, a Matlab-like, free scientific computing package (http://www.scilab.org/). We present here two simulation results for a North Sea net with 100 meshes around, 52 meshes long, $4 \mathrm{~mm}$ twine diameter, $0.05 \mathrm{~m}$ mesh side, using the third order nonlinear law above, placed in a uniform current of $1 \mathrm{~m} / \mathrm{s}$ generating drag and lift forces as explained in section 3.2. The magnitude of the drag force is $0.03 \mathrm{~N}$ per node and the lift force $0.016 \mathrm{~N}$ per node. The computations were performed on a $1.25 \mathrm{GHz}$ Apple PowerBook G4.

The first simulation corresponds to a circular basis $\Gamma$ and the second to an elliptical basis. The opening radius in the circular case is of $0.8 \mathrm{~m}$. In the elliptical case, we just flatten the circle by one half in one direction.

In order to start the conjugate gradient algorithm not too far from the expected equilibrium, we first compute a slightly stretched conical configuration based on the circular configuration shown above, see figure 5.1, to serve as initial configuration.

The algorithm is stopped whenever the relative change of the energy between two successive iterations is less than $10^{-9}$ or when the number of iterations exceeds 20,000 . It should be noted that the dimension of the minimization space, i.e., the number of nodes not on $\Gamma$, is of the order of 10,000 in these simulations.

The circle simulation stops in 5,312 iterations with the equilibrium configuration below (see figures 5.2 and 5.3)

Below in figure 5.4 is a plot of the energy vs. iteration number. It is apparent that the equilibrium was actually reached around 2,500 iterations and that nothing much was achieved after that. Note the large jumps in the energy which correspond to iterations where the Polak-Ribière algorithm finds a particularly good descent direction.

The maximum twine stretch in the equilibrium configuration is of 1.0008911, which is well within the linear regime as expected. 
Figure 5.2: 5312 Iterations

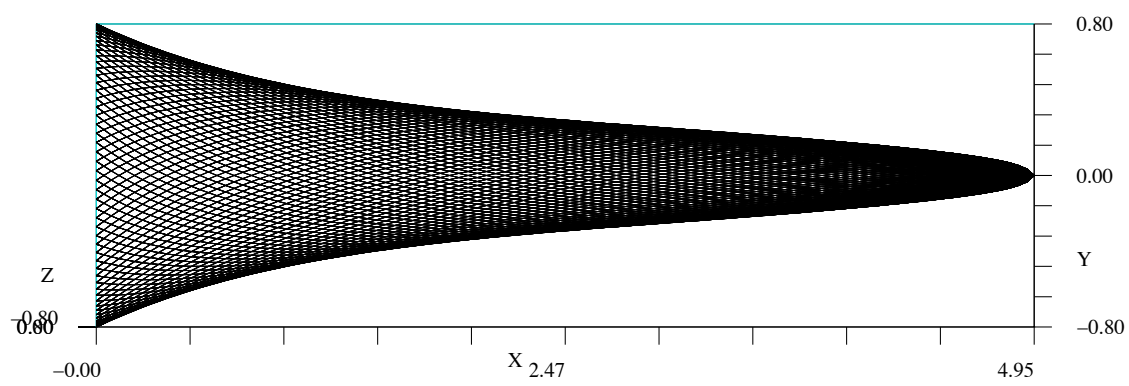

Figure 5.3: 5312 Iterations; view in perspective

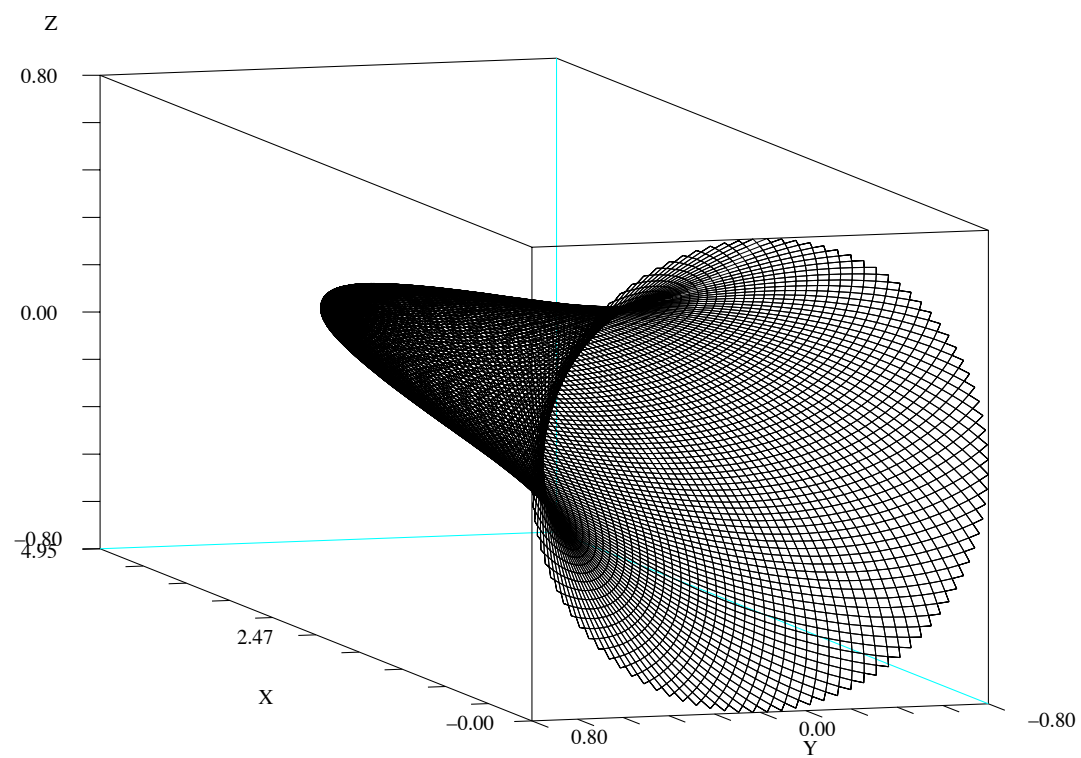

The ellipse simulation runs in 2,408 iterations

The maximum stretch is 1.0371934 .

Il faut peut-être des conclusions... 
Figure 5.4: Eergy decay test

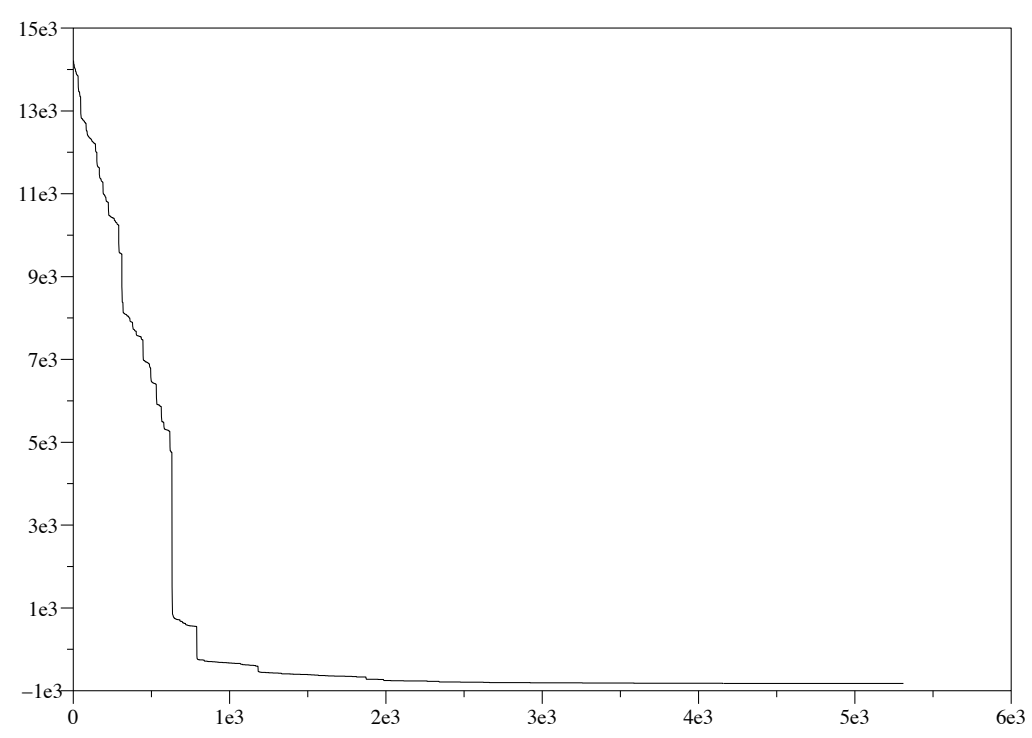

Figure 5.5: Ellipse simulation, topview

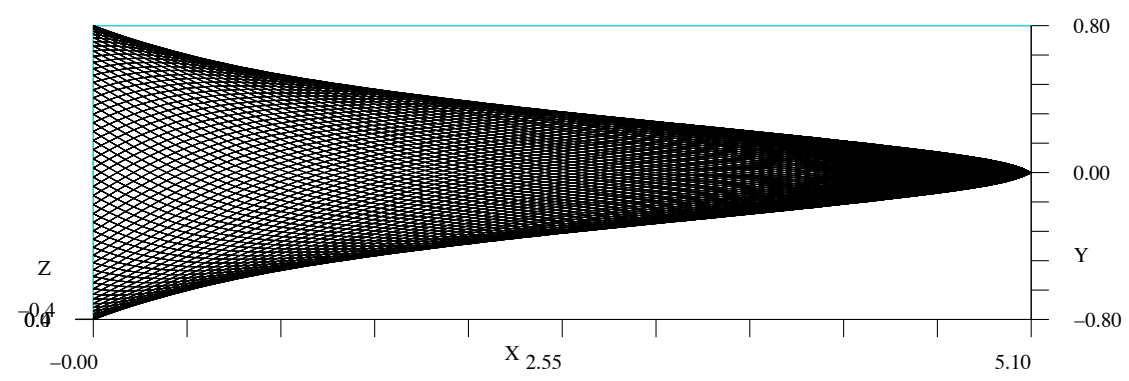

Figure 5.6: Ellipse simulation, sideview

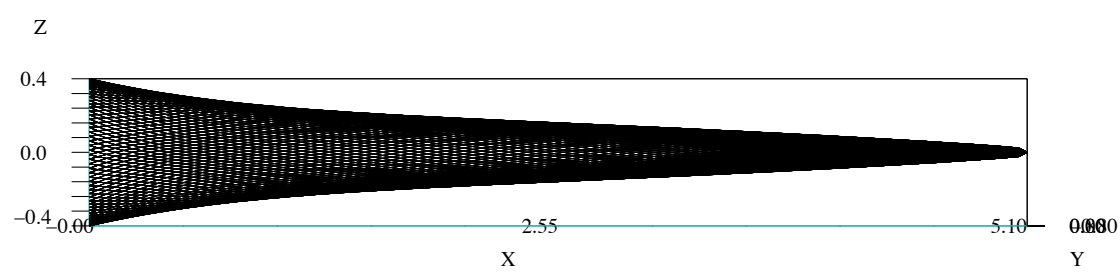


Figure 5.7: Ellipse simulation, perspective view

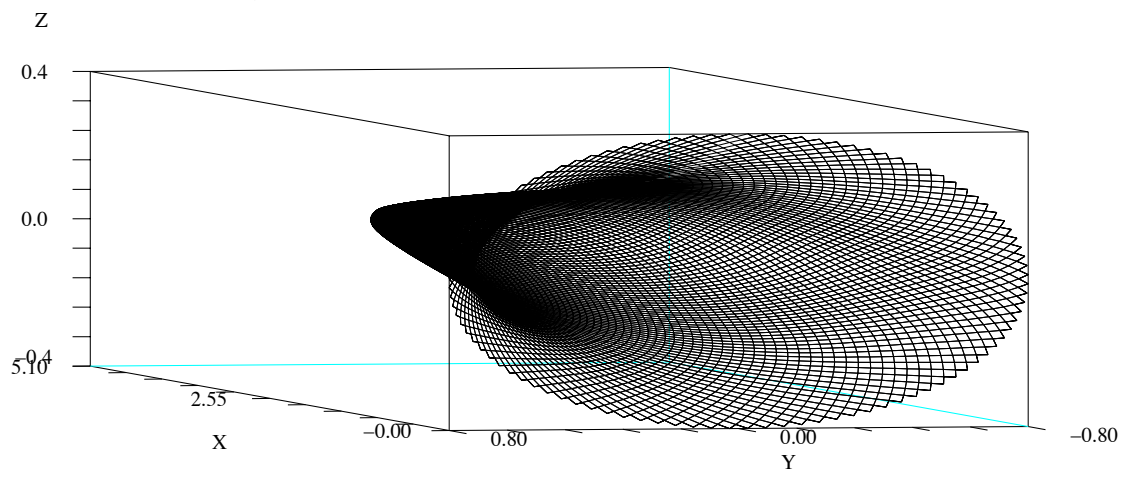

\section{References}

[1] E. Acerbi, G. Buttazzo, and D. Percivale. A variational definition for the strain energy of an elastic string. J. Elasticity, 25:137-148, 1991.

[2] P. L. Bourget and D. Marichal. Remark about variation in the drag coefficient of circular cylinders moving through water. Ocean Engineering, 17:569-585, 1991.

[3] A. Braides and M.S. Gelli. Continuum limits of discrete systems without convexity hypotheses. Math. Mech. Solids, 7:41-66, 2002.

[4] P. G. Ciarlet. Introduction l'Analyse Numérique Matricielle et l'Optimisation. Masson, 1982.

[5] P. G. Ciarlet. Mathematical Elasticity. Volume I: Three-Dimensional Elasticity. North-Holland, Amsterdam, 1988.

[6] W. A. Green and J. Shi. Deformation of discrete elastic networks. IMA Journ. of Appl. Math., 43:99-112, 1990.

[7] W. A. Green and J. Shi. Plane deformations of membranes formed with elastic cords. Q. J. Mech. Appl. Math., 43:318-333, 1990.

[8] O. Pironneau I. Danaila, H. Hecht. Simulation numérique en C++. Dunod, 2003.

[9] L. Landweber and M. H. Plotter. The shape and tension of a light flexible cable in a uniform current. Journal of Applied Mechanics, June:121-126, 1947.

[10] C. Nedelec and G. Portier. Science et pêche. bulletin d'information et de documentation de l'ISTPM, Nantes, 227, 1973.

[11] F. G. O'Neil. Differential equation governing the geometry of a diamond mesh codend of a trawl net. Journal of applied mechanics, 64:7-14, 1997.

[12] F. G. O'Neil. Axisymmetric trawl cod-ends made from netting of a general mesh shape. IMA Journal of Applied Math., 62:245-262, 1999. 
[13] O. Pironneau. Méthode des éléments finis pour les fluides. Masson, rma edition, 1988.

[14] D. Priour. Calculation of net shapes by the finite element method with triangular elements. Comm. in Num. Meth., 15:755-763, 1999.

[15] D. Priour. Introduction of a mesh resistance to opening in a triangular element for calculation of nets by finite elements. Comm. in Num. Meth. in Eng., 17:229-237, 2001.

[16] R. S. Rivlin. Plane strain of a net formed by inextensible cords. Journ. Rat. Mech. Anal., 4:951-974, 1955.

[17] R. S. Rivlin. The deformation of a membrane formed by inextensible cords. Arch. Rat. Mech. Anal., 3:447-476, 1959.

[18] F. Theret. Etude de l'équilibre de surfaces réticulées placées dans un courant uniforme; application aux chaluts. Thèse IFREMER, 1993. 